\title{
Gap Junction Proteins Expressed during Development Are Required for Adult Neural Function in the Drosophila Optic Lamina
}

\author{
Kathryn D. Curtin, Zhan Zhang, and Robert J. Wyman \\ Department of Molecular, Cellular, and Developmental Biology, Yale University, New Haven, Connecticut 06511
}

We provide evidence that gap junction proteins, expressed during development, are necessary for the formation of normally functioning connections in the Drosophila optic lamina. Flies with mutations in the gap junction genes (innexins), shaking $B$, and ogre have normal photoreceptor potentials but a defective response of the postsynaptic cells in the optic lamina. This is indicated by a reduction in, or absence of, transients in the electroretinogram. Ogre is required in the presynaptic retinal photoreceptors. ShakingB $(N)$ is, at a minimum, required in postsynaptic lamina neurons. Transgenic expression of the ap- propriate innexins during pupal development (but not later) rescues connection defects. Transient gap junctions have been observed to precede chemical synapse formation and have been hypothesized to play a role in connectivity and synaptogenesis; however, no causal role has been demonstrated. Here we show that developmental gap junction genes can be required for normally functioning neural connections to form.

Key words: gap junctions; innexins; neural connections; Drosophila; visual system; development
Temporary gap junctions (GJs) have been demonstrated between synaptic partners before synaptogenesis and have been hypothesized to instruct chemical synapse formation. Fischbach (1972) first reported electrical coupling between cultured neurons and their subsequent chemical synaptic partners. GJs form before chemical synapse formation between cultured myotomes and neurons from Xenopus (Peng et al., 1980; Allen and Warner, 1991) and also between cultured motoneurons and myoblasts from early, but not late, stage chick embryos (Bonner, 1989). In Daphnia, GJs lasting a few hours were seen by electron microscopy (EM) between retinal neurons and their later chemical synaptic partners in the optic lobe (Lopresti et al., 1974).

Transient GJs have been implicated in connectivity. GJs may specify functional domains for establishing neural maps by coordinating electrical or biochemical activity between adjacent neurons of a given type before synaptogenesis (Yuste et al., 1995; Kandler and Katz, 1998; Chang and Balice-Gordon, 2000). Temporary GJs may influence pathfinding. The pioneer neuron of the grasshopper limb bud forms transient GJs with guidepost cells (Bentley and Keshishian, 1982). Likewise, segmentally repeating interneurons in the leech form temporary GJs with counterparts in other segments that may lead to growth restriction as well as determine the direction of neural branching (Wolszon et al., 1994, 1995).

Although temporary GJs have been hypothesized to instruct connectivity and synaptogenesis, tools have not been available until recently to demonstrate a causal link. The identification of GJ genes in Drosophila allows experimental perturbation of GJ genes during development. Using molecular genetic techniques, we demonstrate that developmental expression of GJ genes is

\footnotetext{
Received March 12, 2002; revised May 7, 2002; accepted May 13, 2002.

This work was funded by National Institutes of Health Grant GM57889 and by the Charles E. Culpeper Foundation. We thank Yi-An Sun and Haig Keshishian for assistance and support.

Correspondence should be addressed to Kathryn D. Curtin, Department of Molecular, Cellular, and Developmental Biology, 266 Whitney Avenue, New Haven, CT 06511. E-mail: kathryn.curtin@yale.edu.

Copyright (C) 2002 Society for Neuroscience $0270-6474 / 02 / 227088-09 \$ 15.00 / 0$
}

required for normally functioning adult visual transmission. This report focuses on the spatial and temporal requirements of two GJ genes.

In vertebrates, GJs are formed by connexins (Swenson et al., 1989; Kumar and Gilula, 1996; Unger et al., 1999). However, in invertebrates, GJs are formed by the innexin family (Sun and Wyman, 1996; Phelan et al., 1998; Landesman et al., 1999; Stebbings et al., 2000). Mutations in several innexins in Drosophila and Caenorhabditis elegans lead to GJ loss (Phelan et al., 1996, Starich et al., 1996; Sun and Wyman, 1996). For example, mutations in Drosophila that eliminate the neural class of shakingB (shB) proteins, $\operatorname{shB}(\mathrm{N})$ and $\operatorname{shB}(\mathrm{N}+16)$, lead to a loss of GJs between the giant fiber (GF) and its partners (Thomas and Wyman 1984; Krishnan et al., 1993; Phelan et al., 1996; Sun et al., 1996) as well as GJ loss in the haltere system (Trimarchi and Murphey, 1997) and between some larval muscles (Todman et al., 1999). Mutations in the Drosophila innexin gene ogre cause GJ loss in specific tissues (Y. A. Sun and R. J. Wyman, unpublished observations). Null ogre mutants are pupal lethal, whereas hypomorphic mutants have reduced optic ganglia (Watanabe and Kankel, 1990, 1992). Some innexins form homotypic GJs in coupled Xenopus oocytes (Phelan et al., 1998; Landesman et al., 1999), whereas others, including $\operatorname{shB}(\mathrm{N})$ and ogre, do not (Phelan et al., 1996; K. D. Curtin, D. L. Paul, and R. J. Wyman, unpublished observations). Some innexins are known to multimerize with other family members, forming mixed GJs (Stebbings et al., 2000).

\section{MATERIALS AND METHODS}

Mosaics. The method uses the Flipase Recombinase Target (FRT)/flp recombinase system in which the flipase enzyme (flp) is expressed in the eye under the control of repeated elements of a short, eye-specific enhancer of eyeless (ey) promoter.

Cross: FRT 19A, l(1)CL, GMR-hid/FM7; +; ey-Gal4, UAS-flp $\times$ FRT $19 A$, ogre $^{c b 8} \mathrm{~cm} /$ Y. After recombination, cells homozygous for the recessive lethal gene die. Retinal neurons in which recombination has not occurred also die because of the dominant GMR-hid. Only retinal cells homozygous for ogre $^{c b 8}$ live. These cells repopulate the eye and make a nearly normal size eye with only very small bits of wild-type tissue (data not shown). These bits are revealed by their red eye color (ogre ${ }^{c b 8}$ is 
tightly linked to the recessive eye color marker carmine). Retinal cell death occurs at the larval stage, making it problematic to determine whether retinal axon projections are normal at this stage. In addition, there are no markers to distinguish mosaic larvae or to tell mutant from wild-type axons in larvae.

Electroretinograms. Flies were immobilized in a plastic pipette tip with the head protruding from the tapered end. Flies were oriented with one eye facing a fiber optic lamp positioned behind a shutter. Glass capillaries (1 mm diameter, with filament; Precision Instruments M1B100F-4) were pulled and filled with $130 \mathrm{~mm} \mathrm{NaCl}, 50 \mathrm{~mm} \mathrm{KCl}$. The recording electrode was inserted just inside the cornea, and the ground electrode was placed into the back of the head. A Grass stimulator was used to automatically open the shutter to expose flies to the fiber optic light for $4 \mathrm{sec}$. The response was recorded on an oscilloscope, the screen was photographed on Polaroid film, and the pictures were scanned into a computer. Electrodes were tested for resistance, and similar resistance electrodes were used for all data collection.

Drosophila lines. A $4.5 \mathrm{~kb}$ XhoI-HindIII fragment from upstream of the ogre message was cloned into the Gal4 vector pGatB (Brand and Perrimon, 1993). The XhoI site at the $5^{\prime}$ end was the same site as that in the $5^{\prime}$ end of the clone used by Watanabe and Kankel (1990) for ogre rescue. The HindIII site is within the $3^{\prime}$ end just inside the ogre message start site. $S h B^{N 1}$ and ogre cDNAs were amplified by PCR and cloned into pUAST (Brand and Perrimon, 1993). Constructs were introduced into flies by P-element-mediated transformation (Spradling and Rubin, 1982). Sev-Gal4 was from Liqun Luo, GMR-Gal4 was from Matthew Freeman, hs-Gal4 was from Haig Keshishian, Rh1-tau-lacZ was from the Barry Dickson laboratory, and Rh1-Gal4 was from the Charles Zucker laboratory. The elav-Gal4 enhancer trap line, C155, was obtained from Corey Goodman (University of California Berkeley). $S h B^{2}$ and ogre ${ }^{c b 8}$ mutants were recombined with $\mathrm{C} 155$, and this recombinant was used in rescue experiments by crossing to $U A S-s h B(N)$ and $U A S$-ogre lines, respectively. The following lines were obtained from the Drosophila Stock Center at Indian University: (1) FRT19A, (2)UAS-(nuclear)lacZ, and (3) FRT19A, 1(1)CL1, GMR-hid;+; ey-Gal4, UAS-flp. ogre cbs $^{c \text { lines were }}$ obtained from Doug Kankel (Yale University). Ogre ${ }^{c b 8}$ was recombined with this marker (Ashburner, 1989).

Immunohistochemistry. Adult fly heads were fixed in $3 \%$ paraformaldehyde in $5 \times$ phosphate buffer $(5 \times$ PBS without the $\mathrm{NaCl}$ ) for $5 \mathrm{hr}$, washed three times for $10 \mathrm{~min}$ in PBS, incubated overnight in PBS plus $20 \%$ sucrose, and subsequently mounted in TissueTek (Fischer Scientific) and quick frozen by pressurized $\mathrm{CO}_{2}$. Sections $(10-15 \mu \mathrm{m}$ thick) were collected onto slides pretreated with poly-L-lysine (Sigma, St. Louis, MO). Samples were blocked for $1 \mathrm{hr}$ in PBS plus $1 \%$ Triton X-100 and $2 \%$ BSA or normal goat serum (Vector Laboratories) and then incubated overnight in primary antibody in block. Slides were washed three times for $30 \mathrm{~min}$ in PBS plus $0.5 \%$ Tween 20 . Secondary antibody was applied for $1 \mathrm{hr}$. Biotinylated goat anti-rat secondary antibody (Vector Laboratories, BA-4000) was resuspended in $1 \mathrm{ml}$ PBS and diluted 1:200 in block. Slides were washed as before. The Vector ABC HRP kit (Vector Laboratories, PK-6100) or ABC Alkaline Phosphatase kit (see Fig. $4 F$ ) were used according to manufacturer's instructions. Visualization was via the Vector VIP stain (SK4600). Slides were mounted with Permount and photographed with a digital camera. Antibodies were obtained from the following sources: anti- $\beta$-galactosidase (gal) (Promega, Z378A) used 1:1000, and anti-synaptotagmin, from Hugo Bellen (Baylor College of Medicine) (Littleton et al., 1993), used 1:100. Anti-shB antibody was generated in rats by injection of a fusion protein containing the $\mathrm{C}$-terminal tail of the shB protein.

\section{RESULTS}

\section{ShB and ogre mutant animals are defective in electroretinogram transients}

Electroretinograms (ERGs) record the presynaptic receptor potential and the postsynaptic response of the lamina. Figure $1 \mathrm{~A}$ shows an ERG of a wild-type animal. The large sustained corneanegative (downward going) potential (receptor potential) records depolarization of retinal photoreceptors in response to light. This response is independent of synaptic mechanisms (Hotta and Benzer, 1969; Pak et al., 1969; Burg et al., 1993).

A lights-on stimulus induces a rapid cornea-positive (upward) spike (on-transient). This is followed by a cornea-negative rapid

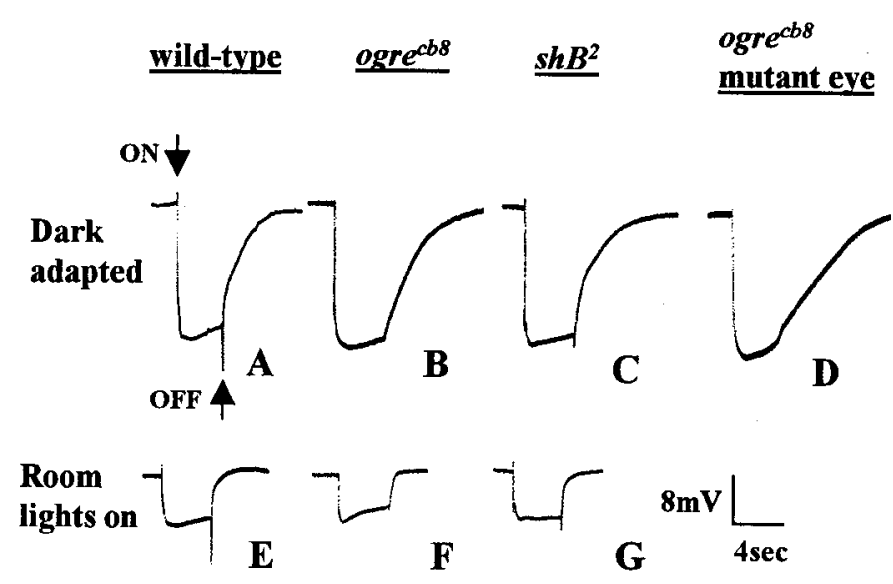

Figure 1. ERGs of wild-type, ogre $e^{c b s}, s h B^{2}$, and ogre ${ }^{c b 8}$ mutant-eye animals. $A, E$, ERGs of wild-type flies after dark adaptation for 15 min $(A)$ or with room lights on $(E)$. A 4 sec bright-light pulse is marked by the arrows in $A$ that demarcate the on- and off-transient. $B, F$, ERGs of ogre $e^{c b 8}$ flies after dark adaptation $(B)$ or with room lights on $(F)$. $C, G$, ERGs of $s h B^{2}$ flies after dark adaptation $(C)$ or with room lights on $(G)$. $D$, ERG of dark-adapted ogre $e^{c b s}$ mutant-eye mosaics.

transient response, which is usually masked by the receptor potential and is therefore not discussed further. When the light is switched off, a cornea-negative transient spike (off-transient) is seen, followed by recovery of the receptor potential. These onand off-transients (Fig. $1 A$, arrows) are postsynaptic responses of the lamina monopolar neurons that the retinal neurons synapse with in the lamina (Alawi and Pak, 1971; Heisenberg, 1971). There are no GJs between retina and lamina neurons in the adult. These are purely chemcial synapses.

The L1 and L2 monopolar neurons are believed to be primarily responsible for generating the ERG transients (Coombe, 1986). Mutations that disrupt neurotransmitter synthesis in the retina (Hotta and Benzer, 1969; Pak et al., 1969; Burg et al., 1993) or eliminate neurotransmitter release (Stowers and Schwarz, 1999) abolish transients but have no effect on the receptor potential. To date, all published mutants that show normal receptor potentials, but are missing transients, have defects in synaptic transmission for one reason or another.

Both $s h B$ and ogre mutants have defects in ERG on- and off-transients (Homyk et al., 1980; Lipshitz and Kankel, 1985). Figure 1 illustrates these defects for ogre ${ }^{c b 8}$, a hypomorphic mutation of ogre and $s h B^{2}$, a nonsense mutation that eliminates both shB neural proteins (Table 1). Ogre ${ }^{c b s}$ flies showed no ERG transients (Fig. 1B), although the magnitude of the receptor potential was completely normal. $\mathrm{S} h B^{2}$ flies had significantly reduced transients: on-transients were $\sim 39 \%$ of wild-type and off-transients were $\sim 23 \%$ of the wild-type transients (Fig. $1 C$, Table 1). The receptor potential of $s h B^{2}$ flies was also normal. The fact that the presynaptic response in $s h B^{2}$ and ogre ${ }^{c b 8}$ flies is normal, but that excitation of the lamina is eliminated (ogre) or greatly reduced $\left(\operatorname{sh} B^{2}\right)$, indicates a loss of functional chemical synaptic transmission between the retinal photoreceptors and lamina monopolar neurons in these animals. Coombe (1986) has shown that small transients, $\sim 20 \%$ of normal, can be seen even after $70 \%$ of the L1 and L2 cells have degraded. Thus the reduced transients in shakingB reflect a serious loss of lamina response.

We recorded ERGs from animals under two different lighting conditions, either dark-adapted for $15 \mathrm{~min}$ (Fig. $1 A-D$, Table 1) or with room lights on as background illumination (Fig. $1 E-G$, 
Table 1. ERG phenotypes of $s h B^{2}$ and $o g r e^{c b 8}$ animals under two lighting conditions

\begin{tabular}{|c|c|c|c|c|c|c|}
\hline \multirow[b]{2}{*}{ Genotype } & \multicolumn{3}{|c|}{ Room lights on } & \multicolumn{3}{|c|}{ Room lights off (dark adapted) } \\
\hline & $\begin{array}{l}\text { On transient } \\
(\mathrm{mV})\end{array}$ & $\begin{array}{l}\text { Off transient } \\
(\mathrm{mV} \pm \mathrm{SD})\end{array}$ & $\begin{array}{l}\text { Receptor potential } \\
(\mathrm{mV} \pm \mathrm{SD})\end{array}$ & $\begin{array}{l}\text { On transient } \\
(\mathrm{mV} \pm \mathrm{SD})\end{array}$ & $\begin{array}{l}\text { Off transient } \\
(\mathrm{mV} \pm \mathrm{SD})\end{array}$ & $\begin{array}{l}\text { Receptor potential } \\
(\mathrm{mV})\end{array}$ \\
\hline Wild type & $2.2 \pm 0.4$ & $8.0 \pm 0.9$ & $9.7 \pm 1.0$ & $2.3 \pm 0.4$ & $7.2 \pm 0.9$ & $23.0 \pm 2.0$ \\
\hline $\operatorname{sh} B^{2}$ & $1.4 \pm 0.2$ & $2.5 \pm 0.4$ & $9.0 \pm 0.9$ & $0.9 \pm 0.5$ & $1.6 \pm 0.4$ & $22.0 \pm 2.5$ \\
\hline ogre $c b 8$ & 0 & 0 & $8.8 \pm 0.7$ & 0 & 0 & $22.5 \pm 2.3$ \\
\hline
\end{tabular}

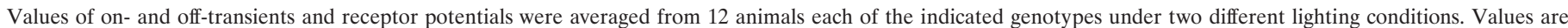

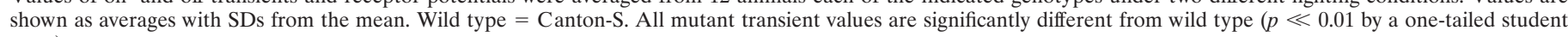
$t$ test).

Table 1). The magnitude of the receptor potential was clearly less with background illumination than in dark-adapted conditions because the fractional change in illumination being detected was less (Fig. 1, compare $A, E$ ). The mutant phenotype of $s h B^{2}$ was slightly more severe when dark-adapted, with both transients $\sim 20 \%$ smaller in dark-adapted animals than with the room lights on (Fig. $1 G$, Table 1). Subsequent rescue experiments with $s h B^{2}$ were done with dark-adapted animals.

For ogre ${ }^{c b 8}$, transients are missing in both lighting conditions; however, we detected partial rescue of the ERG transients (described below) that was most apparent when the room lights were on. We interpret this ability to detect transients more readily when room lights are on as being a result of partial rescue (partial function). This interpretation is supported by the fact that when we increase ogre protein synthesis during rescue by increasing the copy number of the rescue constructs, we see rescue in darkadapted animals as well (data not shown). Because introducing multiple rescue constructs in every experimental situation was not practical, we used the less stringent lights-on conditions to access ogre rescue throughout this study. The ability to detect transients when the room lights are on that are not apparent in dark-adapted animals has also been noted by colleagues in unrelated experimental paradigms in which only partial visual function remains (B. White, personal communication).

\section{Ogre is expressed in a subset of photoreceptor neurons}

Ogre is expressed widely in the embryo and in postembryonic neuroblasts that give rise to the optic ganglia (Watanabe and Kankel, 1992). Ogre message is also detected in the retina of young pupae ( $\sim 14 \mathrm{hr}$ after puparium formation) but not in the adult retina (Watanabe and Kankel, 1992). Last, although ogre message is expressed in the optic formation centers earlier during embryonic and early larval development, this expression ends just after puparium formation (Watanabe and Kankel, 1992).

To determine exactly in which cells in the visual system ogre is expressed, we cloned the ogre promoter next to the Gal4 gene and used it to drive expression from a $U A S$-lac $Z$ gene encoding a nuclear $\beta$-gal protein. The ogre promoter that we cloned included all the upstream sequences $(\sim 4.5 \mathrm{~kb})$ needed for rescue (Watanabe and Kankel, 1990) (see Materials and Methods). Because $\beta$-gal is stable and builds up over time, we could visualize in adult animals expression from earlier stages, although in situ studies indicate that ogre is not detectably expressed in the adult (Watanabe and Kankel, 1992; Z. Zhang and R. J. Wyman, unpublished observations).

The labeling of the distal-most nuclei in the eye suggests expression in some or all of the retinal photoreceptor neurons, R1-6 (Fig. 2A). When we used ogre-promoter-Gal4 to drive tau- $\beta$-gal, which localizes in neural projections, we observed ex-

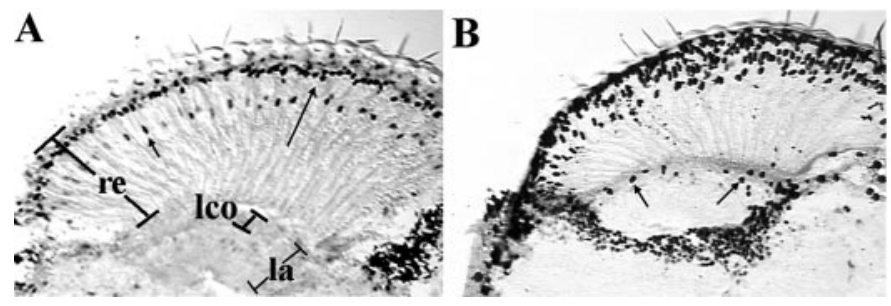

Figure 2. Ogre-promoter-Gal4 and sev-Gal-4 expression patterns in the visual system of adults. Frozen sections of flies with ogre-promoter-Gal4 $(A)$ or sev-Gal4 $(B)$ driving expression of nuclear $\beta$-gal visualized by immunohistochemistry. In $A$, the long arrow in the retina marks the more distal layer of some or all of R1-6 nuclei. The short arrow in the retina marks the more proximal nuclei belonging to $\mathrm{R} 7$. There is no expression in the lamina cortex, marked by brackets, where the cell bodies of the postsynaptic neurons reside. Lco, Lamina cortex; re, retina; la, lamina. In $B$, sev-Gal4-expressing eye cells include R3, R4, R7, and cone cells. $\mathrm{Sev}-\mathrm{Gal} 4$ is also expressed in cells at the retina-lamina margin (arrows) as well as in many outside the optic lamina.

pression in the retinal neuron endings in the lamina, confirming that expression was in some or all of R1-6 (data not shown). The more proximal nuclei in the eye are from R7. There was no expression in R8. There also seemed to be a very low level of expression in the lamina but not in the monopolar neurons from which the transients arise (Fig. 2A) (monopolar neurons are in the lamina cortex) (Meinertzhagen and Hanson, 1993). This entire staining pattern was apparent by mid-pupal development (data not shown), but we saw no retinal expression in larvae. This general pattern and timing of expression in the retina are in agreement with previously published pupal in situ studies (Watanabe and Kankel, 1992).

\section{Both $\operatorname{sh} B(N)$ and ogre have a neural focus of activity}

To begin to determine where $\operatorname{sh}(\mathrm{N})$ and ogre protein are required, we used the Gal4/UAS system to drive expression of $\mathrm{shB}(\mathrm{N})$ (or ogre) protein in specific cell types in $s h B^{2}$ (or ogre $e^{c b 8}$ ) mutants [Brand and Perrimon (1993) describe the Gal4/UAS system]. Expression of $\operatorname{sh} B(N)$ via elav-Gal4, which expresses in all neurons from neural birth through adulthood, caused nearly complete rescue of the $s h B^{2}$ ERG phenotype [(Fig. 3D) compare with wild-type (Fig. $1 A$ ) and $\operatorname{sh} B^{2}$ (Fig. 1C)]. The on-transient in the rescued animals was $91 \%$ of the wild-type level compared with $39 \%$ for $s h B^{2}$ with no transgenes. The off-transient in rescued animals was $76 \%$ of wild type compared with $23 \%$ for $\operatorname{sh} B^{2}$ with no transgenes (Table 2$). \mathrm{ShB}(\mathrm{N}+16)$, which is identical to $\operatorname{shB}(\mathrm{N})$ except that it has an additional 16 amino acids at the $\mathrm{N}$ terminus, rescued the ERG phenotype with similar efficiency to $\operatorname{shB}(\mathrm{N})$ (data not shown). Expression of ogre via elav-Gal4 in ogre ${ }^{c b 8}$ flies mediated partial rescue with on-transients that are $57 \%$ of wild type and off-transients that were $56 \%$ of wild type 


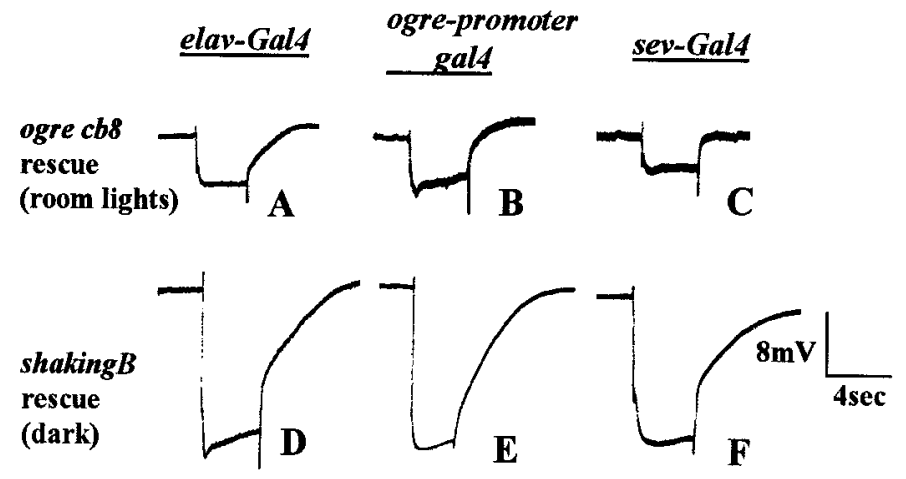

Figure 3. Rescue of the ERG phenotypes of $s h B^{2}$ and ogre $e^{c b 8}$ using various Gal4 drivers. $A-C$, ERGs from ogrec ${ }^{c b s}$ males in which ogre protein is driven by elav-Gal4 $(A)$, ogre-promoter-Gal4 $(B)$, or sev-Gal4 $(C)$. All were tested with room lights on. Compare with wild type (Fig. 1D) and ogre $^{c b 8}$ (Fig. 1E). All three drivers lead to partial rescue. $D-F$, ERGs of $s h B^{2}$ males in which $\operatorname{shB}(\mathrm{N})$ expression is driven by elav-Gal4 $(D)$, ogre-promoter-Gal4 $(E)$, or sev-Gal4 $(F)$. All were dark adapted. Compare with Figure 1, $A$ and $C$. Only elav-Gal4 rescues completely.

(Fig. 3A, Table 2). These results show that the lack of both $\operatorname{shB}(\mathrm{N})$ and ogre in neurons is responsible for the defects in the visual system.

In addition, although expression of ogre via elav-Gal4 rescued the ERG phenotype, frozen sections of these animals had reduced optic ganglia mostly indistinguishable from ogre ${ }^{c b s}$ without transgenes (Curtin and Wyman, unpublished observations), presumably because expression comes on too late to rescue the proliferation of neuroblasts in the optic formation centers. This shows that the ERG phenotype may be rescued mostly independently of the optic ganglia phenotype.

\section{Does removing ogre from the eye disrupt retina-lamina communication?}

Ogre is expressed in photoreceptors and required in neurons. This suggests that it may be required in photoreceptors. To determine this, we tested the ERGs of mosaic flies in which the eye is completely mutant for ogre, and all other body tissues, including the lamina, are heterozygous (phenotypically wild type) for ogre. Such mosaics were generated by the method of Stowers and Schwarz (1999) (see Materials and Methods for a description of the cross). ERGs from ogre $e^{c b s}$ mutant-eye flies were completely lacking on- and off-transients when dark adapted [(Fig. 1D) eight flies tested with same result] and had very small transients when tested with the room lights on (on-transient $=0.39 \pm 0.2 \mathrm{mV}$; off-transient $=0.97 \pm 0.4 \mathrm{mV}$; average of eight animals). We obtained the same results using ogre ${ }^{j N L 3}$ (Watanabe and Kankel, 1990), a null allele (data not shown; rare viable males were used for the cross). Control mosaics generated with a nonmutant chromosome were normal (data not shown).

Last, these mosaic animals differ from ogre ${ }^{c b 8}$ mutants in having normal-size optic ganglia. Staining of head sections from ogre-mutant eye animals with the neural specific anti-elav antibody was indistinguishable from wild type (data not shown), showing an apparently normal complement of cells in the optic ganglia. Figure $4, D$ and $E$, also shows that ogre-mutant eye animals have a normal-size optic ganglia.

\section{Can expression of ogre in the eye restore retina-lamina connections?}

Ogre was required in photoreceptors for lamina neurons to respond normally to photoreceptor depolarization. To determine whether expression in photoreceptors was sufficient to rescue the ERG phenotype of ogre $e^{c b s}$, we used several retina-specific drivers to drive ogre protein expression.

(1) ogre-promoter-Gal4 was expressed in a subset of retinal neurons as described. This expression was evident by mid-pupal stages. There was no apparent expression in lamina neurons (Fig. 2A) (Meinertzhagen and Hanson, 1993).

(2) The sevenless (sev)-Gal4 gene is expressed in retinal neurons 3, 4, and 7, as well as in cone cells (Tomlinson et al., 1987) beginning before the third larval instar. To determine where this driver expresses later in development, we used it to drive nuclear $\beta$-gal and examined frozen sections of adult heads (Fig. $2 B$ ). We observed expression in the eye, including R7 as well as cells, possibly glia, at the margin between the retina and lamina. There is no obvious expression in postsynaptic lamina neurons (Meinertzhagen and Hanson, 1993).

(3) GMR-Gal4 expresses in retinal neurons $1-8$ commencing in the larval stages.

(4) Rhodopsin 1 (Rh1)-Gal4 expresses in retinal neurons 1-6 beginning late in pupal development.

Expression of ogre via the ogre-promoter-Gal4 driver rescued the ERG phenotype (Fig. 3B, Table 2) with on-transients that were $61 \%$ of wild type and off-transients that were $72 \%$ of wild

Table 2. Rescue of $\operatorname{shB}^{2}$ and $o g r e^{c b s}$ ERGs with various Gal4 drivers

\begin{tabular}{|c|c|c|c|c|c|c|}
\hline & \multicolumn{3}{|c|}{$s h B^{2}$ rescue (dark-adapted) } & \multicolumn{3}{|c|}{ ogre $^{c b 8}$ rescue (room lights on) } \\
\hline & $\begin{array}{l}\text { On transient } \\
(\mathrm{mV})\end{array}$ & $\begin{array}{l}\text { Off transient } \\
(\mathrm{mV})\end{array}$ & $\begin{array}{l}\text { Receptor potential } \\
(\mathrm{mV})\end{array}$ & $\begin{array}{l}\text { On transient } \\
(\mathrm{mV})\end{array}$ & $\begin{array}{l}\text { Off transient } \\
(\mathrm{mV})\end{array}$ & $\begin{array}{l}\text { Receptor potential } \\
(\mathrm{mV})\end{array}$ \\
\hline Wild type ${ }^{* *}$ & $2.3 \pm 0.4$ & $7.2 \pm 0.9$ & $23.0 \pm 2.0$ & $2.2 \pm 0.4$ & $8.0 \pm 0.9$ & $9.7 \pm 1.0$ \\
\hline No driver & $0.9 \pm 0.5$ & $1.7 \pm 0.4$ & $22.5 \pm 2.3$ & 0 & 0 & $8.8 \pm 0.7$ \\
\hline GMR-Gal4 & $0.8 \pm 0.6$ & $1.8 \pm 0.3$ & $25 \pm 0.5$ & ND & ND & ND \\
\hline Rh1-Gal4 & $1.0 \pm 0.6$ & $1.8 \pm 0.5$ & $24.0 \pm 3.0$ & 0 & 0 & $8.7 \pm 1$ \\
\hline Ogre-pro-Gal4 & $1.2 \pm 0.4$ & $2.0 \pm 0.3$ & $23.0 \pm 2.0$ & $1.4 \pm 0.5$ & $5.2 \pm 0.8$ & $9.3 \pm 2.0$ \\
\hline sev-Gal4 & $1.7 \pm 0.7^{*}$ & $0.95 \pm 0.2$ & $23.0 \pm 2.0$ & $1.5 \pm 0.5$ & $4.2 \pm 0.7$ & $8.0 \pm 0.9$ \\
\hline Elav-Gal4 & $2.1 \pm 0.3^{*}$ & $5.5 \pm 0.5^{*}$ & $24 \pm 1.5$ & $1.3 \pm 0.2$ & $4.0 \pm 0.5$ & $9.3 \pm 1$ \\
\hline
\end{tabular}

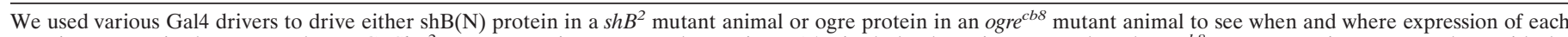

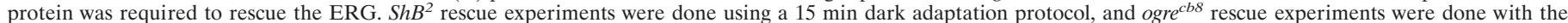

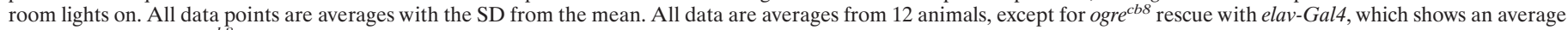
of 8 animals, and ogre $e^{c b 8}$ rescue with sev-Gal4, which is an average of 6 animals. ND, Not done.

* Statistically significant rescue for $s h B^{2}$.

**Wild-type values repeated from Table 1 . 

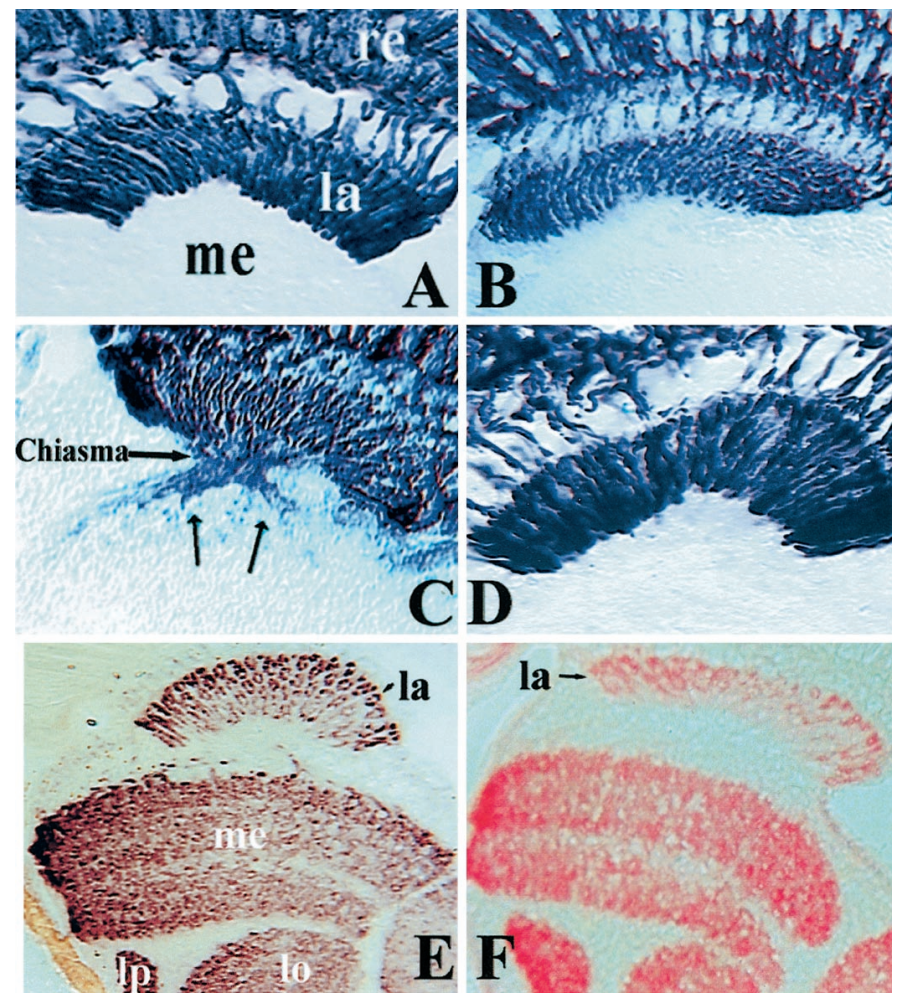

Figure 4. Retinal projections and localization of synaptic markers are relatively normal in $s h B^{2}$ and $o g r e^{c b s}$ mutant eye animals. R1-6 projections in $s h B^{2}$ mutants and mosaics with ogre ${ }^{c b 8}$ mutant eyes are mostly normal. Frozen sections of wild-type adult heads $(A), \operatorname{sh} B^{2}$ males $(B, C)$, and ogre ${ }^{c b 8}(D)$ mutant-eye mosaics, all expressing Rh1-tau-lacZ, which expresses $\beta$-gal in R1-6. $B$, Approximately $76 \%$ of $s h B^{2}$ flies show normal projections. $C$, Approximately $24 \%$ of $s h B^{2}$ flies show abnormal projections. Arrows in $C$ mark R1-6 axons that have passed through the lamina. $E$, Frozen head sections of ogre $e^{c b 8}$ mutant-eye mosaics stained with anti-synaptotagmin. Synaptotagmin localization is indistinguishable from wild-type (data not shown) or $s h B^{2}$ flies (data not shown). F, Frozen head sections of flies in which $\operatorname{shB}(\mathrm{N})$ is expressed in all neurons via the elav-Gal4 driver. Sections were stained with anti-shB(N) antibody. la, Lamina; me, medulla; lo, lobula; $l p$, lobula plate.

type, compared with no transients in ogre ${ }^{c b 8}$ alone. Rescue was seen consistently only when the room lights were on. Rescue improved to nearly complete with an increased number of copies of ogre-promoter-Gal4 and UAS-ogre (data not shown), suggesting that partial rescue was a result of inadequate levels of protein expression. Attempts to rescue the ogre ${ }^{c b 8}$ ERG phenotype via the sev-Gal4 driver led to similar partial rescue with on-transients that were $65 \%$ of wild type and off-transients that were $58 \%$ of wild type (Fig. 3C, Table 2). If expression was driven with both ogre-promoter-Gal4 and sev-Gal4 together, we observed nearly wild-type transients (see Table 4).

Expression of ogre via the GMR-Gal4 line, which expresses at high levels in all retinal neurons, led to severe eye roughness (data not shown), indicating retinal cell death or failure of some retinal cells to develop (Tomlinson et al., 1987; Wolff and Ready, 1992). The significant loss of retinal cells made this driver unsuitable for testing rescue of the ERG.

Ogre-promoter-Gal4 and sev-Gal4 rescued the transients, and both are expressed in retinal photoreceptors. The only overlap in the staining pattern of these two drivers is in the retina, and combined use of both drivers led to complete rescue (see Table 4, top row). This leads us to conclude that ogre expression in a subset of retinal neurons may be sufficient for functioning retinalamina connections to form.

Last, although expression of ogre via sev-Gal4 partially rescued the ERG phenotype, frozen head sections of these animals showed that the optic ganglia are mostly indistinguishable from ogre $^{c b 8}$ with no transgenes, as was also the case with elav-Gal4 ERG rescue animals (data not shown). Thus ERGs can be rescued even in animals with defective optic ganglia. The small optic ganglia of ogre mutants is a result of a defect in cell proliferation, and possibly cell death, in the optic formation centers early in development (Lipshitz and Kankel, 1985), whereas retina-lamina connections form later during pupal development when ogre is expressed in the retina (Watanabe and Kankel, 1992).

\section{Where is $\operatorname{sh} B(N)$ expression needed?}

Expression of $\operatorname{shB}(\mathrm{N})$ in both presynaptic and postsynaptic cells via elav-Gal4 rescues the ERG (Table 2). Pupal in situ studies show that $\operatorname{sh} B$ is expressed in the lamina and retinal neurons peaking at the mid-pupal stage (Crompton et al., 1995), whereas in the adult, $\operatorname{shB}(\mathrm{N})$ and $\operatorname{shB}(\mathrm{N}+16)$ are expressed in the monopolar neurons in the adult lamina but not in the retina (Zhang et al., 1999). Thus, $\operatorname{shB}(\mathrm{N})$ may be required in both presynaptic and postsynaptic cells or in just one of these cell types.

To determine whether retinal expression of $\operatorname{shB}(\mathrm{N})$ is sufficient, we used the same retinal drivers for ERG rescue. Expression of $\mathrm{shB}(\mathrm{N})$ via retina-expressing drivers, including the ogre-promoterGal4 line (Fig. 3E), the GMR-Gal4 line, and the Rh1-Gal4 line did not rescue the $s h B^{2}$ ERG phenotype (Table 2). The sev-Gal4 driver did not rescue the off-transient of $\operatorname{sh} B^{2}$, although there was weak rescue of the on-transient (Fig. $3 F$, Table 2) (see Discussion). When we used these same retinal drivers to express $\operatorname{sh}(\mathrm{N})$ in a wild-type background, we saw normal ERGs. This shows that the negative rescue results were not caused by exogenous $\operatorname{sh}(\mathrm{N})$ interfering with endogenous innexins.

Expression of $\operatorname{shB}(\mathrm{N})$ in both presynaptic and postsynaptic cells via the elav-Gal4 enhancer traps rescued completely, whereas expression in the presynaptic cells alone gave no rescue or partial rescue. This shows that $\operatorname{shB}(\mathrm{N})$ is needed postsynaptically in the lamina for important aspects of its function (see Discussion). At the moment, tools are not available to pursue the cellular requirements of $\operatorname{shB}(\mathrm{N})$ as far as we could for ogre. We cannot make eye mosaics because $\operatorname{sh} B$ lies closer to the centromere than any of the available FRT insertions. In addition, no lamina-specific drivers have been characterized to allow us to determine whether $\operatorname{shB}(\mathrm{N})$ expression in the lamina is sufficient for rescue.

\section{Expression is required during development}

To determine when $\operatorname{shB}(\mathrm{N})$ is needed, we used $h s-G a l 4$ to drive expression at different times in $\operatorname{sh} B^{2}$ mutants. This heat-shock driver has low-level expression at a low temperature $\left(17^{\circ} \mathrm{C}\right)$ and increased expression at a high temperature $\left(31^{\circ} \mathrm{C}\right)$. We tested three groups of animals. A control group was kept continuously at the low-expressing temperature and tested 4-5 d after hatching. A second group was heat shocked to the high-expressing temperature from the white prepupal stage through approximately twothirds of pupation. These flies were then placed at the lowexpressing temperature and tested 4-5 d after hatching. A third group was heat shocked for $2 \mathrm{~d}$ as adults and tested immediately after heat shock.

Mutants that were heat shocked only as adults were indistin- 


\begin{tabular}{|c|c|c|}
\hline \multicolumn{3}{|c|}{$\begin{array}{l}\text { Table 3. Determining when } \operatorname{shB}(\mathrm{N}) \text { is needed to rescue the } \operatorname{sh} B^{2} \\
\text { ERG phenotype }\end{array}$} \\
\hline & On transient $(\mathrm{mV})$ & Off transient $(\mathrm{mV})$ \\
\hline No heat shock & $2.3 \pm 0.2$ & $1.8 \pm 0.4$ \\
\hline Heat shock (development) & $2.8 \pm 0.4$ & $4.3 \pm 0.4$ \\
\hline Heat shock (adult) & $2.0 \pm 0.5$ & $1.6 \pm 0.6$ \\
\hline
\end{tabular}

$\overline{\text { Expression of } \operatorname{shB}(\mathrm{N}) \text { was driven by a heat-shock promoter in a } \operatorname{sh} B^{2} \text { mutant animal }}$ at various times during development to determine when the protein is needed to rescue the ERG phenotype. Data shown are averages from eight flies with the SD shown. The first row is data from flies not subjected to heat shock. The second row is from flies heat shocked from late in the third larval instar through late pupal development. The third row is from flies that were heat shocked only as adults. Receptor potentials were unaffected by heat shock.

guishable from flies that were never heat shocked (Table 3), showing that adult expression alone is insufficient for rescue. The on-transient was rescued by $h s$-Gal4 even at the low-expressing temperature (Table 3). This might be attributable to leaky expression (see also Discussion). Animals reared at the lowexpressing $17^{\circ} \mathrm{C}$ showed no rescue of off-transients; they were indistinguishable from $s h B^{2}$ flies without transgenes (Table 3; compare Table 1, wt, dark-adapted). However, animals heat shocked during the first two-thirds of pupal development showed significant rescue of off-transients to $60 \%$ of wild-type values. This represents a $2.7 \times$ increase over the off-transient values for $s h B^{2}$ without rescue constructs and is comparable to rescue seen with elav-Gal4. In addition, these animals showed rescue $5 \mathrm{~d}$ after heat shock was terminated. Because preliminary experiments show that innexins have a half-life of $\sim 6 \mathrm{hr}$ in the animal, similar to that seen with connexins (Fallon and Goodenough, 1981; Zhang and Wyman, unpublished observations), this suggests that $\operatorname{shB}(\mathrm{N})$ may not be needed in the adult. The time window during which $\operatorname{shB}(\mathrm{N})$ was required includes the developmental stage when retinal axons rearrange and chemical synapses form.

We could not use heat shock to determine when ogre is needed, because expression of ogre via $h s-G a l 4$ was lethal to ogre $e^{c b s}$ animals, which have very low viability even without heat shock. However, on the basis of several pieces of data, ogre expression is required during development. First, ogre message can be detected in the visual system of early pupae but not in adults (Watanabe and Kankel, 1992). Second, the sev-Gal4 and elav-Gal4 drivers, which mediated ERG rescue of ogre ${ }^{c b 8}$, begin expression by the third larval instar. Third, ogre-promoter-Gal4 driver expressed in the retina by the mid-pupal stage (data not shown). Fourth, the R1-6 expressing driver, Rh1-Gal4, which turns on in the last half of pupal development after chemical synapse formation is complete (Meinertzhagen and Hanson, 1993), does not rescue (Table 2). Rh1-Gal4 showed high levels of $\beta$-gal expression in R1-6 (data not shown). Last, below we describe ogre phenotypes that implicate ogre in late, pupal stages of development of retina-lamina connections (also see Discussion).

\section{Do ogre and $\operatorname{shB}(\mathrm{N})$ affect R1-6 pathfinding?}

R1-6 in shB $^{2}$ animals

Normally the axons for R1-6 terminate in the lamina, whereas $\mathrm{R} 7$ and $\mathrm{R} 8$ axons pass through the lamina to terminate in the medulla, with R8 terminating distally to the central brain and R7 proximally. In pathfinding mutants, errors are usually evident by the third larval instar (Garrity et al., 1999). We examined 55 third larval instar nervous systems of $\operatorname{sh} B^{2}$ animals: in 20 animals all retinal axons were stained with chaoptin antibody (24B10) (Fujita et al., 1982) and 35 animals were marked with Ro-lacZ (Garrity et al., 1999), which expresses in R2-5, and stained with an anti- $\beta$-gal antibody. No retinal projection defects were seen at this stage with either method.

We examined R1-6 projections in adult $s h B^{2}$ flies by marking them with an Rh1-tau lacZ construct (Newsome et al., 2000) and staining frozen adult head sections with an anti- $\beta$-gal antibody. Wild-type R1-6 projections are shown in Figure $4 A$. In $\operatorname{sh} B^{2}$ adults, R1-6 appeared normal in most animals (Fig. 4B), although axons are so tightly packed in the lamina that subtle disorganization would not be easily detectable. In some flies (6 of 25 ), a fraction of R1-R6 neurons extended beyond the lamina and into the first optic chiasm (Fig. 4C). These defects were rescued by expression of $\operatorname{shB}(\mathrm{N})$ in all neurons via elav-Gal4 (data not shown). Because R1-6 apparently successfully find and stop in the lamina in the larval stage, it may be later during pupation that some mutant axons extend beyond the lamina. The results suggest that pathfinding is mostly normal in $s h B^{2}$ animals.

\section{R1-6 in animals with ogre ${ }^{c b 8}$ mutant eyes}

We cannot examine pathfinding at the third larval instar of ogre mutants because the optic ganglia are defective. For technical reasons, we also cannot interpret ogre mutant-eye mosaics at this stage (see Materials and Methods). To examine R1-R6 in adults with ogre $e^{c b 8}$ all-mutant eyes, we made mosaics that carried an Rh1-tau-lacZ marker on the second chromosome. The axons stopped correctly in the lamina and did not exhibit obvious pathfinding errors (Fig. $4 D$ ), although in $\sim 10 \%$ of samples we saw occasional small gaps in the staining pattern of R1-6 (data not shown). Control mosaics generated by the same method were indistinguishable from wild type (data not shown).

\section{Normal localization of synaptic proteins}

Although the synaptic connections are not functional, at least one synaptic protein localizes normally. Using an antibody to synaptotagmin (Littleton et al., 1993), we found that synaptotagmin distribution was found in a wild-type pattern in both $\operatorname{sh} B^{2}$ (data not shown) and ogre ${ }^{c b 8}$ mutant-eye animals (Fig. $4 E$ ). The normal localization of synaptotagmin in $\operatorname{sh} B^{2}$ is consistent with EM data showing the presence of synaptic vesicles (Shimohogashi and Meinertzhagen, 1998) (see Discussion). We do not have a good antibody to ogre, and $\operatorname{shB}(\mathrm{N})$ is not detectably expressed in the adult or developing visual system with our current antibody. However, when we overexpressed $\operatorname{shB}(\mathrm{N})$ in adult heads via elav-Gal4, we observed localization to brain neuropile in a pattern grossly similar to synaptotagmin (Fig. $4 F$ ). Expression of a myc-tagged ogre protein in all neurons via elav-Gal4 showed the same pattern of staining as that shown for $\operatorname{shB}(\mathrm{N})$ (data not shown). In addition, myc-tagged versions of both proteins localize to the cell membrane in a punctate staining pattern when expressed in the salivary gland (data not shown), consistent with their established roles as GJ proteins.

\section{Between what cells are gap junctions forming?}

Developmental GJs could form between the photoreceptor neurons of the retina and the neurons of the lamina. An alternative is that GJs form within the retina and within the lamina, but not between the two; i.e., ogre is a component of intra-retinal GJs and $\operatorname{shB}(\mathrm{N})$ is a component of intra-lamina GJs. Neither ogre nor $\operatorname{shB}(\mathrm{N})$ is able to form homotypic GJs in a number of assays (Phelan et al., 1996; Curtin and Paul, unpublished observations; Sun and Wyman, unpublished observations). Thus, no matter where they participate in GJ formation, they probably need to mate with other innexins to form GJs. 


\begin{tabular}{llcl}
\hline $\begin{array}{l}\text { Table 4. Can ogre } \\
\text { junctions in the eye? }\end{array}$ & ERGs be rescued by making homotypic gap \\
& $\begin{array}{l}\text { On transient } \\
(\mathrm{mV})\end{array}$ & $\begin{array}{l}\text { Off transient } \\
(\mathrm{mV})\end{array}$ & $\begin{array}{l}\text { Receptor } \\
\text { potential }(\mathrm{mV})\end{array}$ \\
\hline Ogre expressed & $2.7 \pm 0.8$ & $8.2 \pm 2.2$ & $8.9 \pm 0.5$ \\
shB(L) expressed & $0.8 \pm 0.8$ & $0.16 \pm 0.2$ & $9.0 \pm 0.4$ \\
Wild-type control* $^{*}$ & $2.2 \pm 0.4$ & $8.0 \pm 0.9$ & $9.7 \pm 1.0$ \\
\hline
\end{tabular}

Expression of ogre (top row) or shB(L) (second row) was driven in an ogre ${ }^{c b 8}$ animal using a combination of sev-Gal4 and ogre-promoter-Gal4. Expression of ogre rescues (first row) and expression of $\operatorname{shB}(\mathrm{L})$ does not (second row). Data were averaged from eight animals of each genotype. Averages are shown with the SD.

*Wild-type values from Table 1.

If the function of ogre is to take part in intra-retinal junctions, then it should be possible to rescue ogre ERGs by expressing $\mathrm{shB}(\mathrm{L})$, a homotypic innexin protein (Krishnan et al., 1995; Phelan et al., 1998), in the eye, thus restoring intra-retinal GJs. $\mathrm{ShB}(\mathrm{L})$ was expressed in the retinal neurons of ogre ${ }^{c b 8}$ mutants using ogre-promoter-Gal4 and sev-Gal4 together. This is the promoter combination that effected complete rescue when driving ogre (Table 4). When driving $\operatorname{shB}(\mathrm{L})$, however, there was no appreciable rescue of the ERG transients (Table 4).

We cannot test whether expression of $\operatorname{shB}(\mathrm{L})$ in both presynaptic and postsynaptic cells would rescue ogre, because expression via the elav-Gal4 driver leads to lethality (Stebbings et al., 2000; K. D. Curtin and R. J. Wyman, unpublished observations), and no more specific driver that expresses in retina and lamina is available.

\section{DISCUSSION}

Transient GJs have been observed in developing nervous systems throughout the animal kingdom, including between cells that will later form chemical synaptic pairs (see introductory remarks). Although developmental GJs have been hypothesized to affect target selection and synaptogenesis, no causal relationship has been established previously. Here we show that developmentally expressed GJ proteins are required for normally functioning adult connections to form in the Drosophila optic lamina. $S h B^{2}$ and ogre-mutant eye animals have ERG defects indicative of failure of the lamina monopolar neurons to respond to their presynaptic partners, the retinal photoreceptors. Ogre is required in the retina, and $\operatorname{shB}(\mathrm{N})$ is required, at a minimum, in the lamina. These genes are expressed during pupal development when final connections are forming, and transgenic expression during pupal development is required to rescue the connections. Development of the presynaptic or postsynaptic tissue appears normal through the third larval instar by several criteria. These genes are required for a late step in development of functional connections in the optic lamina.

\section{Normal development of retina-lamina connections}

Connectivity between R1-6 and the lamina occurs in two distinct phases. During the third larval instar, R1-6 terminate in the lamina plexus in a regular pattern with R1-6 axons from a single ommatidium remaining together in a fascicle and $\mathrm{R}$ cells from adjacent ommatidia terminating in adjacent fascicles. No chemical synapses are formed at this stage. During early pupal development, $\sim 30 \mathrm{hr}$ after $\mathrm{R} 1-6$ reach the lamina, the retinal axons in each ommatidium defasciculate and extend growth cones across the lamina surface. Rearranging R1-6 growth cones make stereotypical contacts with each other before terminating at and forming synapses with their final lamina targets. The adult lamina is composed of repeating units (cartridges) containing several monopolar neurons (L1-5), as well as R1-6 axons. Final wiring occurs so that each cartridge receives inputs from one of each R1-6 neuron. Each of these R cells come from different ommatidia, and all point in the same direction; i.e., they are stimulated by the same bit of the visual field. R1-6 synapse primarily with monopolar neurons and amacrine cells, and chemical synapse formation is complete by mid-pupal development (Meinertzhagen and Hanson, 1993).

\section{Multiple neural circuits in the optic lamina}

The on- and off-transients arise from lamina monopolar neurons (Alawi and Pak, 1971; Heisenberg, 1971; Coombe, 1986). The on-transient is caused primarily by the monosynaptic connections from photoreceptors (Meinertzhagen and O'Neil, 1991; Burg et al., 1993). $\mathrm{ShB}(\mathrm{N})$ seems to be required for these connections to form normally. The on-transient is significantly affected in $\operatorname{sh} B^{2}$ (Table 1) and in a fraction of $s h B^{2}$ animals; photoreceptor axons pass by the lamina (Fig. 4C).

The off-transient includes a contribution from a second pathway. This may be a disynaptic pathway from retina to amacrine cells to lamina monopolars (Meinertzhagen and O'Neil, 1991). The amacrine-monopolar synapses may be cholinergic because cholinergic synapses, not coming from photoreceptors, contribute to the off-transient (Greenspan et al., 1980).

$\mathrm{ShB}(\mathrm{N})$ may also be required for the establishment of this pathway. $\operatorname{ShB}(\mathrm{N})$ transgenic expression sometimes rescued the on-transient but not the off-transient (Table 3, top line). $\mathrm{ShB}(\mathrm{N})$ could be required in the eye to promote retinal to lamina monopolar connections that contribute to the on-transient. For its role in generating the off-transient, however, $\operatorname{shB}(\mathrm{N})$ could be required in amacrine neurons to promote retina to amacrine connections or in amacrine or lamina monopolar neurons to promote connections between these two cell types. Ogre affects both transients and thus could contribute to both pathways. Other innexins may contribute to the development of either pathway. One is expressed in the lamina (Curtin et al., 1999), and several have not been characterized. Given the complexity of the final chemical synaptic connections, any required developmental GJ connections might be quite complex.

\section{Innexins act during early pupal development}

We believe that ogre and $\operatorname{sh}(\mathrm{N})$ act primarily during the pupal stage when photoreceptor axons rearrange to find and synapse with their final lamina targets. First, both genes are expressed in the visual system during the first half of pupal development (Watanabe and Kankel, 1992; Crompton et al., 1995). Although $\operatorname{sh} B$ message is also detected in the adult lamina (Z hang et al., 1999), ogre is not detected in adults (Watanabe and Kankel, 1992; Zhang and Wyman, unpublished observations). Intra-lamina or retina-lamina GJs have not been observed in the adult, so the function of $\operatorname{shB}(N)$ in the adult lamina is unclear. Second, both genes were required sometime between late larval mid-pupal stages for rescue (see Results). Third, R1-6 axons of $s h B^{2}$ and ogre $^{c b 8}$ mutant-eye animals stopped normally in the lamina in early larval stages.

Last, the photoreceptor projections to the lamina in the third larval instar induce a final division and differentiation of lamina target cells (Meinertzhagen and Hanson, 1993). This occurs normally in $s h B^{2}$, which contains the normal complement of cells in lamina cartridges (Shimohogashi and Meinertzhagen, 1998). Similarly, ogre $e^{c b s}$ mutant-eye mosaics showed a normal arrangement 
of neural cells in the optic lamina via anti-elav staining (data not shown). All of these observations argue for normal retina-lamina contacts through the third larval instar. This places the developmental disruption in the early pupal period when these genes are expressed in the retina or lamina (Watanabe and Kankel, 1992; Crompton et al., 1995) and when the retinal photoreceptor axons are reorganizing and making their final synaptic connections.

In addition to a developmental function, either protein may have an adult function. In wild-type animals, retinal axons are coupled very sparsely by GJs in the adult. This coupling is reduced very slightly but not eliminated in $\operatorname{sh} B^{2}$ (Shimohogashi and Meinertzhagen, 1998). The possible role of ogre in these junctions is unknown. The function of these adult GJs is unknown.

\section{For what developmental process are GJs required?}

$\mathrm{ShB}(\mathrm{N})$ and ogre are not needed for early pathfinding. However, there may be subtle disorganization of R1-6 axons in either $s h B^{2}$ or ogre $e^{c b s}$ mutant-eye animals, which might indicate defective sorting out of axons during early pupae. This may be why $24 \%$ of $s h B^{2}$ adults showed some R1- 6 axons passing the lamina after the larval stage. Future analysis should help us to determine whether axon projections within the lamina are normal for both mutants [via EM or see Clandinin and Zipursky (2001)].

EM analysis of $s h B^{2}$ animals, however, shows that lamina cartridges can be structurally quite normal and include synaptic vesicles and presynaptic ribbons (Shimohogashi and Meinertzhagen, 1998). Future, structural analysis will illuminate this issue for both mutants, but subtle disorganization may not explain the synaptic transmission defects given the very robust nature of the ERG transients (Coombe, 1986).

The role of $\operatorname{shB}(\mathrm{N})$ in pathfinding and synapse formation has been studied in the GF system. The GF forms dual electrical/ chemical synapses with the TTMn jump motoneuron (Blagburn et al., 1999). The electrical synapses are missing in $s h B^{2}$ mutants by electrophysiological and dye-fill criteria (Thomas and Wyman, 1984; Phelan et al., 1996; Sun and Wyman, 1996). In $s h B^{2}$ mutants, the GF contacts its target cells normally (Sun and Wyman, 1996; Jacobs et al., 2000). Synaptic vesicles are also present at the contact point between the GF and the TTMn (Blagburn et al., 1999). It has not been possible to assay the function of GF to TTMn chemical synapses in wild-type animals because their activity would be masked by the more rapidly occurring spike response caused by the electrical synapse. However, electrophysiological data suggest that this chemical synapse functions very weakly in $\operatorname{sh} B$ mutants, failing at stimulation rates of $>1 / \mathrm{sec}$ (Thomas and Wyman, 1984; Baird et al., 1990). In addition, presynaptic machinery can be morphologically normal even in the absence of a normally functioning synapse (Prokop et al., 1996; Allen et al., 1999). Determining the exact defect leading to a failure of information transfer may require in-depth structural and functional studies, which we leave to later research.

\section{Where are gap junctions forming?}

Two nonmutually exclusive possibilities seem most likely. First, ogre could participate in GJs between photoreceptors, whereas $\operatorname{shB}(\mathrm{N})$ participates in GJs between lamina neurons. For example, intra-retinal GJs could form during early pupal development when retinal axons make stereotypical contacts with each other (Meinertzhagen and Hanson, 1993). These contacts may be important for final wiring to be accurate (Clandinin and Zipursky, 2001). Although light-evoked activity is not required for connec- tions to form normally in Drosophila (Meinertzhagen and Hanson, 1993), spontaneous activity has not been examined. Such activity, transmitted through GJs, is important for fine-tuning retinal connections in vertebrates (Penn et al., 1994). Alternatively, ogre and $\operatorname{shB}(\mathrm{N})$ may be components of transient GJs between presynaptic and postsynaptic neurons, as have been observed in Daphnia and other systems (see introductory remarks). Such GJs could easily play a role in final target selection or the formation of a functional chemical synapse.

\section{REFERENCES}

Alawi AA, Pak WL (1971) On-transient of insect electroretinogram: its cellular origin. Science 172:1055-1057.

Allen F, Warner A (1991) Gap junctional communication during neuromuscular junction formation. Neuron 6:101-111.

Allen MJ, Shan X, Caruccio P, Froggett SJ, Moffat KG, Murphey RK (1999) Targeted expression of truncated glued disrupts giant fiber synapse formation in Drosophila. J Neurosci 19:9374-9379.

Ashburner M (1989) Drosophila: a laboratory handbook. Cold Spring Harbor, NY: Cold Spring Harbor Laboratory.

Baird DH, Schalet AP, Wyman RJ (1990) The Passover locus in Drosophila melanogaster: complex complementation and different effects on the giant fiber neural pathway. Genetics 126:1045-1059.

Bentley D, Keshishian H (1982) Pathfinding by peripheral pioneer neurons in grasshoppers. Science 218:1082-1088.

Blagburn JM, Alexopoulos H, Davies JA, Bacon JP (1999) Null mutation in shaking- $B$ eliminates electrical, but not chemical, synapses in the Drosophila giant fiber system: a structural study. J Comp Neurol 22:449-458.

Bonner PH (1989) Correlation of development stage and gap junction formation between chick embryo neurons and cloned skeletal muscle myoblasts. Exp Cell Res 181:205-216.

Brand AH, Perrimon N (1993) Targeted gene expression as a means of altering cell fates and generating dominant phenotypes. Development 118:401-415.

Burg MG, Sarthy PV, Koliantz G, Pak WL (1993) Genetic and molecular identification of a Drosophila histidine decarboxylase gene required in photoreceptor transmitter synthesis. EMBO J 12:911-919.

Chang Q, Balice-Gordon RJ (2000) Gap junctional communication among developing and injured motor neurons. Brain Res Rev 32:242-249.

Clandinin TR, Zipursky SL (2001) Afferent growth cone interactions control synaptic specificity in the Drosophila visual system. Neuron 28:427-436.

Coombe PE (1986) The large monopolar cells L1 and L2 are responsible for ERG transients in Drosophila. J Comp Physiol [A] 159:655-665.

Crompton D, Todman M, Wilkin, Ji S, Davies J (1995) Essential and neural transcripts from the Drosophila shakingB locus are differentially expressed in the embryonic mesoderm and pupal nervous system. Development 170:142-158.

Curtin KD, Zhang Z, Wyman RJ (1999) Drosophila has several genes for gap junction proteins. Gene 232:191-201.

Fallon RF, Goodenough DA (1981) Five-hour half-life of mouse liver gap junction protein. J Cell Biol 90:521-526.

Fischbach GD (1972) Synapse formation between dissociated nerve and muscle cells in low density cell cultures. Dev Biol 28:407-429.

Fujita SC, Zipursky SL, Benzer S, Ferrus A, Shotwell SL (1982) Monoclonal antibodies against the Drosophila nervous system. Proc Natl Acad Sci USA 79:7929-7933.

Garrity PA, Lee C-H, Salecker I, Robertson HC, Desai CJ, Zinn K, Zipursky SL (1999) Retinal axon target selection is regulated by a receptor protein tyrosine phosphatase. Neuron 22:707-717.

Greenspan RJ, Finn JA, Hall JC (1980) Acetylcholinesterase mutants in Drosophila and their effects on the structure and function of the central nervous system. J Comp Neurol 189:741-774.

Heisenberg M (1971) Separation of receptor and lamina potentials in the electroretinogram of normal and mutant Drosophila. J Exp Biol 55:85-100.

Homyk Jr T, Szidonya J, Suzuki DT (1980) Behavioral mutants of Drosophila melanogaster. III. Isolation and mapping of mutations by direct visual observations of behavioral phenotypes. Mol Gen Genet 177:553-565.

Hotta Y, Benzer S (1969) Abnormal electroretinograms in visual mutants of Drosophila. Nature 222:354-356.

Jacobs K, Todman MG, Allen MJ, Davies JA, Bacon JP (2000) Synaptogenesis in the giant-fibre system of Drosophila: interaction of the giant fibre and its major motorneuronal target. Development 127:5203-5222.

Kandler K, Katz LC (1998) Coordination of neuronal activity in developing visual cortex by gap junction-mediated biochemical communication. J Neurosci 18:1419-1427.

Krishnan SN, Frei E, Swain GP, Wyman RJ (1993) Passover: a gene 
required for synaptic connectivity in the giant fiber system of Drosophila. Cell 73:967-977.

Krishnan SN, Frei E, Schalet AP, Wyman RJ (1995) Molecular basis of intracistronic complementation in the Passover locus of Drosophila. Proc Natl Acad Sci USA 92:2021-2025.

Kumar NM, Gilula NB (1996) The gap junction communication channel. Cell 84:381-388.

Landesman Y, White TW, Starich TA, Shaw JE, Goodenough DA, Paul DL (1999) Innexin-3 forms connexin-like intercellular channels. J Cell Sci 112:2391-2396

Lipshitz HD, Kankel DR (1985) Specificity of gene action during central nervous system development in Drosophila melanogaster: analysis of the lethal (1) optic ganglion reduced locus. Dev Biol 108:56-77.

Littleton JT, Bellen HJ, Perin MS (1993) Expression of synaptotagmin in Drosophila reveals transport and localization of synaptic vesicles to the synapse. Development 118:1077-1088.

Lopresti V, Macagno ER, Levinthal C (1974) Structure and development of neuronal connections in isogenic organisms: transient gap junctions between growing optic axons and lamina neuroblasts. Proc Natl Acad Sci USA 71:1098-1102.

Meinertzhagen IA, Hanson TE (1993) The development of the optic lobe. In: The development of Drosophila melanogaster, Vol 2 (Bate M, Zarias A. Martine, eds), pp 1363-1491. Cold Spring Harbor, NY: Cold Spring Harbor Laboratory.

Meinertzhagen IA, O'Neil SD (1991) Synaptic organization of the columnar elements in the lamina of the wild type in Drosophila melanogaster. J Comp Neurol 301:232-263.

Newsome TP, Asling B, Dickson BJ (2000) Analysis of Drosophila photoreceptor axon guidance in eye-specific mosaics. Development $127: 851-860$.

Pak WL, Grossfield J, White NV (1969) Nonphototactic mutants in a study of vision of Drosophila. Nature 222:351-354.

Penn AA, Wong RO, Shatz CJ (1994) Neuronal coupling in the developing mammalian retina. J Neurosci 14:3805-3815.

Peng HB, Nakajima Y, Bridgman PC (1980) Development of the postsynaptic membrane in Xenopus neuromuscular cultures observed by freeze-fracture and thin-section electron microscopy. Brain Res 196:11-31.

Phelan P, Nakagawa M, Wilkin MB, Moffat KG, O'Kane CJ, Davies JA, Bacon JP (1996) Mutations in shaking-B prevent electrical synapse formation in the Drosophila giant fiber system. J Neurosci 16:1101-1113.

Phelan P, Stebbings LA, Baines RA, Bacon JP, Davies JA, Ford C (1998) Drosophila Shaking-B protein forms gap junctions in paired Xenopus oocytes. Nature 391:181-184.

Prokop A, Landgraf ER, Broadie K, Bate M (1996) Presynaptic development at the Drosophila neuromuscular junction: assembly and localization of presynaptic active zones. Neuron 17:617-626.

Shimohogashi M, Meinertzhagen IA (1998) The shaking B gene in Drosophila regulates the number of gap junctions between photoreceptor terminals in the lamina. J Neurobiol 35:105-117.
Spradling AC, Rubin GM (1982) Transposition of cloned P elements into Drosophila germ line chromosomes. Science 218:341-347.

Starich TA, Lee RY, Panzarella C, Avery L, Shaw J (1996) Eat-5 and unc-7 represent a multigene family in Caenorhabditis elegans involved in cell-cell coupling. J Cell Biol 134:537-548.

Stebbings LA, Todman MG, Phelan P, Bacon JP, Davies JA (2000) Two Drosophila innexins are expressed in overlapping domains and cooperate to form gap-junction channels. Mol Biol Cell 11:2459-2770.

Stowers RS, Schwarz TL (1999) A genetic method for generating Drosophila eyes composed exclusively of mitotic clones of a single genotype. Genetics 152:1631-1639.

Sun YA, Wyman RJ (1996) Passover eliminates gap junctional communication between neurons of the giant fiber system in Drosophila. J. Neurobiol 30:340-348.

Swenson KI, Jordan JR, Beyer EC, Paul DL (1989) Formation of gap junctions by expression of connexins in Xenopus oocyte pairs. Cell 57:145-155.

Todman MG, Baines RA, Stebbings LA, Davies JA, Bacon JP (1999) Gap-junctional communication between developing Drosophila muscles is essential for their normal development. Dev Genet 24:57-68.

Tomlinson A, Bowtell DD, Hafen E, Rubin GM (1987) Localization of the sevenless protein, a putative receptor for positional information, in the eye imaginal disc of Drosophila. Cell 51:143-150.

Thomas JB, Wyman RJ (1984) Mutations altering synaptic connectivity between identified neurons in Drosophila. J Neurosci 4:530-538.

Trimarchi JR, Murphey RK (1997) The shaking-B ${ }^{2}$ mutation disrupts electrical synapses in a flight circuit in adult Drosophila. J Neurosci 17:4700-4710.

Unger VM, Kumar NM, Gilula NB, Yeager M (1999) Threedimensional structure of a recombinant gap junction membrane channel. Science 283:1176-1180.

Watanabe T, Kankel DR (1990) Molecular cloning and analysis of l(1)ogre, a locus of Drosophila melanogaster with prominent effects on the postembryonic development of the central nervous system. Genetics 126:1033-1044.

Watanabe T, Kankel DR (1992) The l(1)ogre gene of Drosophila melanogaster is expressed in postembryonic neuroblasts. Dev Biol 152:172-183.

Wolff T, Ready DF (1992) Cell death in normal and rough eye mutants of Drosophila. Development 113:825-839.

Wolszon LR, Gao WQ, Passani MB, Macagno ER (1994) Growth cone "collapse" in vivo: are inhibitory interactions mediated by gap junctions? J Neurosci 14:999-1010.

Wolszon LR, Passani MB, Macagno ER (1995) Interactions during a critical period inhibit bilateral projections in embryonic neurons. J Neurosci 15:1506-1515.

Yuste R, Nelson DA, Rubin WW, Katz LC (1995) Neuronal domains in developing neocortex: mechanisms of coactivation. Neuron 14:7-17.

Zhang Z, Curtin KD, Sun YA, Wyman RJ (1999) Nested transcripts of gap junction gene have distinct expression patterns. J Neurobiol 40: 288-301. 